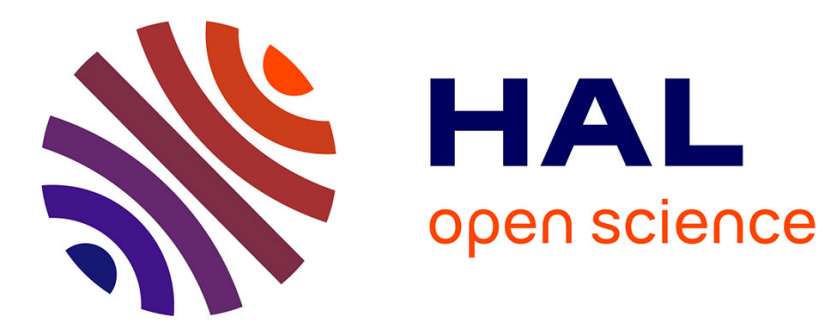

\title{
On the asymptotic theory of localized structures in a thin two-dimensional Harris current sheet: plasmoids, multiplasmoids and $\mathrm{X}$ points
}

\author{
A. Tur, P. Louarn, V. Yanovsky, D. Le Queau, Vincent Génot
}

\section{To cite this version:}

A. Tur, P. Louarn, V. Yanovsky, D. Le Queau, Vincent Génot. On the asymptotic theory of localized structures in a thin two-dimensional Harris current sheet: plasmoids, multiplasmoids and X points. Journal of Plasma Physics, 2001, 66, pp.97-117. 10.1017/S002237780100112X . hal-00306244

HAL Id: hal-00306244

https://hal.science/hal-00306244

Submitted on 23 Sep 2008

HAL is a multi-disciplinary open access archive for the deposit and dissemination of scientific research documents, whether they are published or not. The documents may come from teaching and research institutions in France or abroad, or from public or private research centers.
L'archive ouverte pluridisciplinaire HAL, est destinée au dépôt et à la diffusion de documents scientifiques de niveau recherche, publiés ou non, émanant des établissements d'enseignement et de recherche français ou étrangers, des laboratoires publics ou privés. 


\title{
On the Asymptotic Theory of Localized Structures in Thin 2-D Harris Current Sheet: Plasmoids, Multi-Plasmoids and X-Points
}

\author{
A.TUR, P.LOUARN, V. YANOVSKY* \\ D. Le QUEAU, V.GENOT \\ Centre d'Etude Spatiale des Rayonnements, CNRS, \\ 9, ave du Colonel-Roche B.P. 4346, 31028 Toulouse \\ Cedex 4 FRANCE \\ *Institute for Single Crystals, Nat. Acad. of Sci. \\ Ukraine, Lenine ave. 60 Kharkov 310001, UKRAINE
}

February 20, 2001

\begin{abstract}
We develop a new asymptotic method of resolution of the 2-D equilibrium equation of collisionless plasmas described by the Maxwell-Vlassov equations. This method differs from the classical one proposed by $\mathrm{K}$. Schindler ("Earth's magnetospheric processes, ed. by B. M. McCormac, p.200, D.Reidel, MA,1972) since we consider free-boundary plasmas. Our method is a generalization of usual multiscale asymptotic developments. The first approximation asymptotic solutions are found from the solvability conditions (elimination of increasing and singular terms) in the next approximation. We apply the method to the mathematical description of non linear structures that may form in neutral sheets. Particular solutions describing localized plasmoids (O-point configuration) as well as $\mathrm{X}$-point magnetic configurations are obtained. We also find more general solutions describing a finite number of "magnetic islands" (multiplasmoid solutions) separated by $\mathrm{X}$ points.
\end{abstract}

\section{Introduction}

Thin current layers are common structures in both laboratory and space plasmas. Since they largely contribute to the determination of the geometry of the magnetic field, the question of their mathematical description is central in any problems related to the study of the static state as well as the dynamics of the plasma regions. Among the different types of current layers, those associated to reversals of the magnetic field (neutral sheets) have a particular importance in the physics of the environment of magnetized astrophysical objects. They are 
indeed suspected to display a large variety of dynamical behaviors; the most famous one - the magnetic reconnection - being considered as the standard process of energy releases in natural magnetized plasma. The determination of the structure and the understanding of the dynamics of the neutral sheets have thus been the subjects of a considerable number of experimental, numerical and theoretical studies (see e.g.,Refs [1] - [4] and references therein). A particular emphasis has been put on the identification and the description of the localized structures, such as plasmoids or "magnetic islands", that could inherently result from their dynamics. This is a very active field of space physics since it is generally believed that the formation of plasmoids and of other localized structures (flux ropes, for example) in the terrestrial magnetotail is one of the consequences of substorms, the major form of explosive energy release in the Earth magnetosphere. In the solar corona context, it is also suspected that similar isolated structures could result from the reconfiguration of magnetic arches. Nevertheless, if such structures have been observed by a number of space experiments (see for example [5],[6] and references therein), only a few studies have tackled the problem of their mathematical description. This is the main subject of the present article. We will thus propose a new asymptotic procedure for the resolution of the equilibrium equation of 2-D current sheets that will give useful analytical expressions for describing localized magnetized structures.

In order to precise the originality of this procedure, let us briefly present the existing theories. Using a standard coordinate system (the $x(z)$ axis is parallel (perpendicular) to the layer, $y$ being in the direction of invariance of the system), one obtains the classical 2-D equilibrium equation [7] :

$$
\Delta \Psi=\exp (-\Psi)
$$

(where $\Psi$ is proportional to the vector potential). In the rest of the paper, this formula will be referred as the "Harris" equation. As explained in appendix A, this equation can be derived form either MHD or kinetic considerations, the Grad-Safranov equation being in this particular case formally equivalent to the kinetic equation. Despite their formal equivalence, the two approaches present important physical differences. The kinetic description is based on the Maxwell-Vlassov system of equations and can be considered as exact in collisionless plasmas if the fluctuations are neglected. X-point solutions will thus be physically acceptable. In the framework of ideal MHD alone (without dissipation), the physical validity of such solutions would not be guaranteed.

Concerning the exact results, it has been shown that equation(1) associated with Dirichlet's conditions admits a unique solution in bounded domain [8]. Some particular solutions of equation(1) are also known in the theory of analytical functions (see for example [9]). A family of exact solutions can indeed be obtained from the formula: 


$$
\Psi=-\ln 8 \frac{\left|W^{\prime}(z)\right|^{2}}{\left(1+|W|^{2}\right)^{2}}
$$

(here $z=x+i y$ temporary denotes the complex variable).

This formula was probably proposed for the first time in [10]. Although it looks quite general and seems useful for getting physical interesting solutions, the practical interest of this formula is deceptive. Contrary to what is often supposed, the function $W(z)$ is indeed far from being arbitrary. It must satisfy in any restricted domain of the complex plane the relation :

$$
\Delta \ln \left|W^{\prime}(z)\right|=0
$$

meaning that $W^{\prime}(z)$ has no zero, pole and essential singularity. The function $W^{\prime}(z)$ has thus to be an entire function without zero, which is a very strict limitation. Any functions of this type have the form :

$$
W^{\prime}(z)=\exp (g(z))
$$

where $g(z)$ is an arbitrary entire function. Such functions typically increase at infinity $(|z| \rightarrow \infty)$ as the same velocity as their derivatives (see for example [11]). Thus, generally speaking, equation (2) gives solutions that increase at infinity. They are then not very interesting from a physical point of view. Two known physical solutions are the Bennet pinch and the periodic pinch solutions (see for example,[4]). They correspond to the particular cases: $g(z)=0$ and, $g(z)=z$. Thus, if it is not excluded that other forms for $g(z)$ could be physically interesting, it seems unlikely that formula (2) leads to exact solutions describing localized (non-periodical) structures embedded in the 1-D current sheet.

Let us now consider asymptotic methods of resolution of the equation (1). Looking for solutions verifying: $\frac{\partial}{\partial z} \gg \frac{\partial}{\partial x}$., one can introduce a small parameter $\varepsilon^{2}$ in equation (1) by the scaling :

$$
x \mapsto L_{x} x ; z \mapsto L_{z} z ; \epsilon=\frac{L_{z}}{L_{x}} ; \Psi \rightarrow \Psi+\ln L_{z}^{2} .
$$

The equation (1) then writes:

$$
\partial_{z z}^{2} \Psi+\varepsilon^{2} \partial_{x x}^{2} \Psi=\exp (-\Psi)
$$

An asymptotic scheme for this equation was proposed by K. Shindler [12]. It is presented in details in [13] and widely used in works concerning the Earth 
magnetotail [12] - [18]. K. Shindler uses a multiscale development of the equation (5) by introducing a slow variable $\varepsilon x$ in the bounded $(z, x)$ area. Starting from the $\varepsilon=0$ solution of equation (5) (the well-known 1-D Harris sheet):

$$
\Psi_{0}=-\ln \frac{2 c_{0}^{2}}{\cosh ^{2} c_{0} z}
$$

Schindler considers $c_{0}$ as an arbitrary function of the slow variable $x: c_{0}(x)$. This type of development is valid if the upper and lower plasma boundaries (on the two sides of the neutral sheet) have a fixed form. These boundaries are defined by the zero order approximation function given by (6) supposed to be fixed at all orders of the perturbation theory. A priori given plasma boundaries thus entirely determine the zero approximation solution $c_{0}(x)$. This method is particularly useful if the plasma boundaries can be determined at once as it is the case in laboratory plasmas. In natural plasmas, one cannot know and fix the form of the plasma boundaries in advance. This motivates the development of a new asymptotic method for solving equation (5) in a free boundaries case.

\section{New Asymptotic method for solving the "Har- ris" equation}

\subsection{Difficulties of the direct asymptotic development}

In order to understand the difficulty of the direct asymptotic development of equation (5), let us analyze the origin of its non-uniformities (see for example [19]). Parts of this present analysis will be used later in the development of the regularized asymptotic method.

In order to find solutions embedded in Harris sheet, let us make the following change of variable:

$$
\Psi=-\ln \frac{2 c^{2}(z, x)}{\cosh ^{2} z c(z, x)}
$$

where $c(x, z)$ is a new unknown function. One notes that if $c(x, z) \rightarrow$ const. for $x \rightarrow \pm \infty$, the solution will be localized in a Harris sheet. This change of variable is formally exact. Nevertheless, in the frame of a theory of perturbations, $c(x, z)$ cannot be a completely arbitrary function. It must have no zero or singularity in the domain of interest. In the rest of the paper, we will consider that the function $c(x, z)$ satisfies this property. Note also that if the function $c(x, z)=c_{0}(x)$ is a priori given and if the boundaries of the domain are defined by the equation: $\Psi=$ const., one goes back to Schindler's development.

Substituting formula (7) in (5), one gets the following equation for the function $c(z, x)$ : 


$$
\left(\frac{2 z c+\sinh 2 z c}{\cosh ^{2} z c}\right) \frac{\partial c}{\partial z}-\frac{\partial}{\partial z}\left[\frac{2}{c}(1-z c \tanh z c)\right] \frac{\partial c}{\partial z}=-\varepsilon^{2} \frac{\partial^{2} \Psi}{\partial x^{2}}
$$

where $\frac{\partial^{2} \Psi}{\partial x^{2}}$ writes :

$$
\frac{\partial^{2} \Psi}{\partial x^{2}}=\frac{2}{c^{2}}\left(1+\frac{(z c)^{2}}{\cosh ^{2} z c}\right)\left(\frac{\partial c}{\partial x}\right)^{2}-\frac{2}{c}(1-z c \tanh z c) \frac{\partial^{2} c}{\partial x^{2}}
$$

Introducing the two new functions :

$$
\begin{gathered}
W \equiv \frac{2}{c}(1-z c \tanh z c) \frac{\partial c}{\partial z} \\
F=\frac{c(2 z c+\sinh 2 z c)}{2 \cosh ^{2} z c(1-z c \tanh z c)}
\end{gathered}
$$

(8) takes the form:

$$
-\frac{\partial W}{\partial z}+F W=-\varepsilon^{2} \frac{\partial^{2} \Psi}{\partial x^{2}}
$$

The solution of (12) may be written in the form of a formal non-linear integrodifferential equation :

$$
c=c_{0}+\varepsilon^{2} \int_{0}^{z} d z \frac{c \exp \left(\int F d z\right)}{2(1-z c \tanh z c)} \int d z \frac{\partial^{2} \Psi}{\partial x^{2}} \exp \left(-\int F d z\right)
$$

Let us note here that if $\varepsilon^{2}=0, c_{0}$ may be a function of $x: c_{0}=c_{0}(x)$. Writing the direct asymptotic development as:

$$
c=c_{0}(x)+\sum_{(n)} \varepsilon^{2 n} c_{2 n}(x, z)
$$

substituting it in equation (13) and writing $\Psi_{0}=\frac{2}{C_{0}}\left(1-z c_{0} \tanh z c_{0}\right)$ so that:

$$
F_{0}=-\frac{\partial}{\partial z} \ln \Psi_{0} \equiv F\left(c_{0}\right) .
$$

one observes that the integral in the exponent becomes simple. The development takes the form: 


$$
c=c_{0}(x)-\varepsilon^{2} \int_{0}^{z} \frac{d z^{\prime}}{\left(1-z^{\prime} c_{0} \tanh z^{\prime} c_{0}\right)^{2}} \int_{0}^{z^{\prime}} d z^{\prime \prime}\left(1-z^{\prime \prime} c_{0} \tanh z^{\prime \prime} c_{0}\right)^{2}+\cdots
$$

Let us analyze this expression in the domain:

$$
\left|z c_{0}\right| \lesssim 1
$$

The integrals in equation (16) cannot be calculated explicitly. This is, nevertheless, not necessary for illustrating the role of the singularities. In the domain defined by (17), one has approximately :

$$
1-z c_{0} \tanh z c_{0} \approx 1-\left(z c_{0}\right)^{2} .
$$

Taking into account this approximation, we can easily calculate the integrals in (16). One thus obtains:

$$
c=c_{0}(x)-\frac{\varepsilon^{2}}{c_{0}^{2}(x)}\left\{\frac{4}{15} \frac{1}{1-\left(z c_{0}\right)^{2}}-\frac{2}{15} \ln \left[1-\left(z c_{0}\right)^{2}\right]+\frac{\left(z c_{0}\right)^{2}}{10}-\frac{4}{15}\right\}+\cdots
$$

This equation clearly illustrates the problems resulting from the simple direct development. The most singular term in (19) writes:

$$
c=c_{0}(x)-\frac{\varepsilon^{2}}{c_{0}^{2}(x)} \frac{4}{15} \frac{1}{1-\left(z c_{0}\right)^{2}}+\cdots
$$

The direct development thus gets non valid if $1-\left(z c_{0}\right)^{2} \sim \varepsilon^{2}$. The domain of validity is then restricted to a very thin layer centered on the $z=0$ plane (corresponding to the condition: $1-\left(z c_{0}\right)^{2} \gg \varepsilon^{2}$ or $\left.\left(z c_{0}\right)^{2} \ll 1\right)$. This is clearly not appropriated to the description of 2-D structures presenting some thickness. Our task will then be to construct another asymptotic development of (8) that remains correct when $1-\left(z c_{0}\right)^{2} \gtrsim \varepsilon^{2}$.

Let us consider this question more explicitly. When $1-\left(z c_{0}\right)^{2}=0$ (or, more precisely, when $\left.1-\left(z c_{0}\right) \tanh z c_{0}=0\right)$, the equation that defines the plasma boundaries no more depends on $z$. The boundaries are flat and coincide with those defined in the case of 1-D Harris layer. The general result concerning the unicity of the solution of the "Harris" equation (see introduction) thus states 
that the unique possible solution within these flat boundaries must be the 1D Harris layer. The perturbations must then be zero which is only possible if $\varepsilon=0$. It is then logical that by approaching the boundary the direct asymptotic development gets non-uniform. Another way to understand this difficulty is to refer to the topology of the magnetic field. Isolated magnetic structures correspond to $2-\mathrm{D}$ vector fields in the domain $1-\left(z c_{0}\right) \tanh z c_{0}>0$ that have a non-trivial topology (i.e. presenting elliptic and hyperbolic singular points). The transition of these 2-D fields to a 1-D vector field in the domain defined by the boundaries $1-\left(z c_{0}\right) \tanh z c_{0}=0$ is impossible in the frame of a theory of perturbations because it changes the topology. However, we will see that it is possible to construct an asymptotic development that remains correct when $1-\left(z c_{0}\right)^{2} \geqslant 0$.

Moreover, one notes that if $c_{0}(x)$ decreases with the increasing of $|x|$, then the next term in the development increases even more rapidly. This source of non-uniformity in the direct development is connected to the existence of nonzero solutions for the linear operator in the left part of equation (8). Denoting this operator $\mathcal{L}\left\{c_{0}\right\}$, the equation

$$
\mathcal{L}\left\{c_{0}\right\} c_{(1)}=0
$$

has indeed, in general, non-zero solutions. They will always lead to increasing terms in the asymptotic development. In fixed boundary situations, the problem is formulated in such a way that this difficulty does not appear. It is the main difference between the two situations. As it is now demonstrated, it is nevertheless possible to eliminate the increasing terms. This puts new constraints on the function $c_{0}(x)$.

\subsection{Regularization of the asymptotic development by a generalized multiscale method}

Let us look for a solution $c$ in the form of an asymptotic development where the ascending terms systematically appear :

$$
c=c_{0}+\varepsilon^{2} c_{2}\left(x, \xi_{1}\right)+\varepsilon^{4} c_{4}\left(x, \xi_{1}, \xi_{2}, \widetilde{\xi_{2}}\right)+\cdots
$$

here, $c_{0}=$ const $\sim 1$ and: $\xi_{1}, \xi_{2}, \tilde{\xi_{2}}$ are new variables:

$$
\begin{aligned}
& \xi_{1}=\varepsilon^{2} F_{1}(z)+B_{1} ; B_{1}=\text { Const. } \sim 1 . \\
& \xi_{2}=\varepsilon^{4} F_{2}\left(z, x, \xi_{1}(z)\right)+B_{2} ; B_{2}=\text { Const. } \sim 1 . \\
& \widetilde{\xi_{2}}=\varepsilon^{4} \tilde{F}_{2}\left(z, x, \xi_{1}(z), \xi_{2}\left(z, x, \xi_{1}(z)\right)\right)+\widetilde{B_{2}} ; \tilde{B}_{2}=\text { Const. } \sim 1 .
\end{aligned}
$$


The functions $F_{1}, F_{2}, \widetilde{F}_{2}$ are not known. They have to be determined from the elimination of the singular terms. This method is a generalization of the usual multiscale developments. It is used in the general theory of the asymptotic developments of partial differential equations (see for example [20]).

Using (23), the derivative $\frac{\partial}{\partial z}$ writes :

$$
\begin{aligned}
\frac{\partial}{\partial z} \rightarrow & \frac{\partial}{\partial z}+\varepsilon^{2} \frac{d F_{1}(z)}{d z} \frac{\partial}{\partial \xi_{1}}+\varepsilon^{4}\left(\frac{\partial F_{2}}{\partial z}+\varepsilon^{2} \frac{\partial F_{2}}{\partial \xi_{1}} \frac{d F_{1}(z)}{d z}\right) \frac{\partial}{\partial \xi_{2}}+ \\
& +\varepsilon^{4}\left(\frac{\partial \tilde{F}_{2}}{\partial z}+\varepsilon^{2} \frac{d F_{1}(z)}{d z} \frac{\partial \tilde{F}_{2}}{\partial \xi_{1}}+\varepsilon^{4} \frac{\partial F_{2}}{\partial z} \frac{\partial \tilde{F}_{2}}{\partial \xi_{2}}+\varepsilon^{6} \frac{\partial \widetilde{F}_{2}}{\partial \xi_{2}} \frac{\partial F_{2}}{\partial \xi_{1}} \frac{d F_{1}(z)}{d z}\right) \frac{\partial}{\partial \tilde{\xi}_{2}}+(24) \\
= & \frac{\partial}{\partial z}+\varepsilon^{2} F_{1}^{\prime}(z) \frac{\partial}{\partial \xi_{1}}+\varepsilon^{4} \frac{\partial F_{2}}{\partial z} \frac{\partial}{\partial \xi_{2}}+\varepsilon^{4} \frac{\partial \tilde{F}_{2}}{\partial z} \frac{\partial}{\partial \tilde{\xi}_{2}}+\varepsilon^{6}(\cdots)+\cdots
\end{aligned}
$$

We now substitute the asymptotic development (22) and expression for $\frac{\partial}{\partial z}(24)$ in equation (8). Assuming that the development (22) is an asymptotic one, we can also develop the non-linear coefficients in equation(8) into asymptotic series in $\varepsilon^{2}$. As a result, the following formal series is obtained:

$$
\sum_{(n)} \varepsilon^{2 n} \mathcal{L}_{(2 n)}=0
$$

A sufficient condition for satisfying this equation at the order $n$ is that all coefficients before the terms $\varepsilon^{2 n}$ are nul. This condition will give the equations for the functions $c_{2}, c_{4}, \ldots$. Then, it will be necessary to verify that (22) is indeed an asymptotic development.

Let us now apply this procedure. We note :

$$
\begin{aligned}
f_{0} & =-\frac{2}{c_{0}}\left(1-z c_{0} \tanh z c_{0}\right), \\
\frac{d f_{0}}{d z} & \equiv f_{0 z}^{\prime}=\frac{2 z c_{0}+\sinh 2 z c_{0}}{\cosh ^{2} z c_{0}}
\end{aligned}
$$

The first non-trivial equation is obtained from the condition of nullity of the coefficient for $\varepsilon^{4}$ (corresponding to : $\mathcal{L}_{(4)}=0$ ). This gives :

$$
-\left(\frac{2 f_{0 z}^{\prime}}{f_{0}} F_{1}^{\prime}+F_{1}^{\prime \prime}\right) \frac{\partial c_{2}}{\partial \xi_{1}}=\frac{\partial^{2} c_{2}}{\partial x^{2}}
$$

Let us choose the function $F_{1}(z)$ so that :

$$
\frac{2 f_{0 z}^{\prime}}{f_{0}} F_{1}^{\prime}+F_{1}^{\prime \prime}=-\lambda^{2}
$$


which guarantees the elimination of the singularities in the operator $\mathcal{L}_{(4)}=$ 0 . Putting without lack of generality $\lambda=1, F_{1}$ can be found from equation (27)

:

$$
F_{1}(z)=-\int \frac{d z}{f_{0}^{2}} \int f_{0}^{2} d z
$$

This integral has already been calculated approximately in chapter 2.1 (see equation (19)). Keeping in $F_{1}(z)$ the most singular term only:

$$
F_{1}(z) \simeq-\frac{1}{c_{0}^{2}} \frac{4}{15} \frac{1}{1-\left(z c_{0}\right)^{2}}
$$

$\xi_{1}$ writes:

$$
\xi_{1}=-\frac{\varepsilon^{2}}{c_{0}^{2}} \frac{4}{15} \frac{1}{1-\left(z c_{0}\right)^{2}}+B_{1}
$$

When $\left(z c_{0}\right)^{2} \ll 1, \xi_{1}$ turns to be the usual slow variable (shifted by the $B_{1}$ constant).

Next, from equation (26) we obtain :

$$
\frac{\partial c_{2}}{\partial \xi_{1}}=\frac{\partial^{2} c_{2}}{\partial x^{2}}
$$

A known solution for (31) is :

$$
c_{2}=\frac{M_{0}}{2 \sqrt{\xi_{1}}} \exp \left(-\frac{x^{2}}{4 \xi_{1}}\right)
$$

It is nevertheless clear that(31) admits a broader class of solutions writing :

$$
c_{2}=\frac{1}{2 \sqrt{\pi \xi_{1}}} \int_{-\infty}^{+\infty} u\left(x^{\prime}\right) \exp \left[-\frac{\left(x-x^{\prime}\right)^{2}}{4 \xi_{1}}\right] d x^{\prime}
$$

However, any arbitrary functions $u\left(x^{\prime}\right)$ cannot be used in equation(33). For example, if $u\left(x^{\prime}\right)$ is a polynom or a function that increases as $x^{\prime n}$ when $x^{\prime} \rightarrow$ $\infty$, then the equation(33) gives increasing solutions in the first approximation. Here, we will only consider in details the simplest solution and some of its generalization. For the more general cases, it would be necessary to apply the same procedure than explained below and so to verify that it is possible 
to eliminate the increasing terms in next approximations. This would lead to rather cumbersome calculations.

Let us consider here the simplest case. $c$ takes an explicit form :

$c=c_{0}+\varepsilon^{2} \frac{M_{0}}{2}\left(B_{1}-\frac{\varepsilon^{2}}{c_{0}^{2}} \frac{4}{15} \frac{1}{1-\left(z c_{0}\right)^{2}}\right)^{-\frac{1}{2}} \exp \left[-\frac{x^{2}}{4}\left(B_{1}-\frac{\varepsilon^{2}}{c_{0}^{2}} \frac{4}{15} \frac{1}{1-\left(z c_{0}\right)^{2}}\right)^{-1}\right]$

The difference between the direct development (20) and the present development(34) is now clearly apparent. We already saw that the development (20) is not applicable if $1-\left|z c_{0}\right|^{2} \sim \varepsilon^{2}$. Conversely, the development(34) is applicable with the broader condition :

$$
1-\left|z c_{0}\right|^{2} \gtrsim \varepsilon^{2}
$$

We have also to impose the following condition for $B_{1}$ and $C_{0}$ :

$$
\xi_{10}=B_{1}-\frac{4}{15 c_{0}^{2}} \gtrsim 1
$$

In the domain $\left|z c_{0}\right| \ll 1$, the equation (34) becomes simpler :

$$
c=c_{0}+\varepsilon^{2} \frac{M_{0}}{2 \sqrt{B_{1}}} \exp \left(-\frac{x^{2}}{4 B_{1}}\right)+\cdots
$$

The important point concerning equation (37) comes from the fact that it is not only applicable for small $z$. Its condition of applicability is indeed:

$$
|z| \leq \frac{1}{c_{0}} \sqrt{1-\frac{4}{15 c_{0}^{2}}}
$$

as deduced from expression (30) or (34). Thus, for $c_{0}=1$, the domain of applicability is $|z| \leq 0,856 \approx 0,9 \sim 1$. Note that in this domain one has also the possibility to use the more simple equation (37).

The central question is now to check the validity of the asymptotic development(22). To this end, one has to verify that the variables $\xi_{2}, \widetilde{\xi}_{2}$ and the function $M_{0}\left(\xi_{2}, \widetilde{\xi}_{2}\right)$ can be found in a way that eliminates the increasing terms in the next approximation. Let us here mention that unlike simpler problems, a unique $\xi_{2}$ function is not sufficient to eliminate the increasing terms. In order to find $\xi_{2}, \widetilde{\xi}_{2}$ and $M_{0}\left(\xi_{2}, \widetilde{\xi}_{2}\right)$, it is necessary to study the development of equation (8) up to $\varepsilon^{6}$. This procedure is quite cumbersome and described in Appendix B. The result is: 


$$
c_{2}= \pm \frac{A}{2 \sqrt{\xi_{1}}} \frac{\exp \left(-B_{2}-Q(z, x)\right)}{R}
$$

where:

$$
\begin{gathered}
R=D+(\mp) \frac{\varepsilon^{4} \exp \left(-B_{2}\right)}{15^{2} c_{0}^{2}} \frac{\left[1+\left(z c_{0}\right)^{2}\right]}{\left[1-\left(z c_{0}\right)^{2}\right]^{2}}\left(\frac{x^{2}}{2 \xi_{1}}-1\right)\left(1+\frac{\left(z c_{0}\right)^{2}}{\operatorname{coth}^{2} z c_{0}}\right) \exp (-Q(z, x)) \\
Q(z, x)=\frac{3 \varepsilon^{4}}{15^{2} c_{0}^{4} \xi_{1}^{2}} \frac{\left[1+\left(z c_{0}\right)^{2}\right]}{\left[1-\left(z c_{0}\right)^{2}\right]^{2}}+ \\
+\frac{x^{2}}{4 \xi_{1}}\left\{1-\frac{12 \varepsilon^{4}}{15^{2} c_{0}^{4} \xi_{1}^{2}} \frac{\left[1+\left(z c_{0}\right)^{2}\right]}{\left[1-\left(z c_{0}\right)^{2}\right]^{2}}\right\}+ \\
+\frac{x^{4}}{4 \xi_{1}^{2}} \frac{\varepsilon^{4}}{15^{2} c_{0}^{2} \xi_{1}^{2}} \frac{\left[1+\left(z c_{0}\right)^{2}\right]}{\left[1-\left(z c_{0}\right)^{2}\right]^{2}}
\end{gathered}
$$

Equations (39)- (41) are applicable for $\left|z c_{0}\right|^{2} \lesssim 1-\varepsilon^{2}$. Note also that two signs $( \pm)$ are possible in (39). This is an unexpected new feature of this type of development. As discussed below, each sign will correspond to a different topology of the magnetic field lines ( $\mathrm{X}$ or $\mathrm{O}$ type singular points).

Let us now study with more details the limit of applicability of equations (39) and (40), i.e. when $\left|z c_{0}\right|^{2} \lesssim 1-\varepsilon^{2}$. As earlier, we consider that $c_{0} \gtrsim 1$, and $\xi_{10} \gtrsim 1,(36)$. Let us minimize the expression in brackets in equation (41).

$$
\min \left\{1-\frac{12 \varepsilon^{4}}{15^{2} c_{0}^{4} \xi_{1}^{2}} \frac{\left[1+\left(z c_{0}\right)^{2}\right]}{\left[1-\left(z c_{0}\right)^{2}\right]^{2}}\right\} \approx 1-\frac{24}{15^{2} c_{0}^{4} \xi_{10}^{2}} \approx 0.89 .
$$

Thus, in the limit of applicability of equations (39)- (41), the decreasing of $c_{0}(x, z)$ with respect to $x$ is slower. One indeed gets approximately: $\exp \left(-\frac{x^{2} 0.89}{4 \xi_{1}}\right)$ instead of: $\exp \left(-\frac{x^{2}}{4 \xi_{1}}\right)$. This means that for $z \lesssim 0,89 \approx 1$, the decreasing : $\exp \left(-\frac{x^{2}\left(1-\varepsilon^{4}\right)}{4 \xi_{1}}\right)$ is defined by the same equation (27). The minimum value of the denominator in equation (39) then remains close to 1 . As a result, the limitation for $\mathrm{D}$ is : 


$$
D \mp \frac{4}{15} \exp \left(-B_{2}\right) \gtrsim 1
$$

However, even with values of $z$ such that $z \lesssim 0,93 \approx 1$, the additional term in the denominator (39) is small as compared to $\mathrm{D}$ and can be neglected. Thus, in the domain $z \lesssim 0,89 \approx 1$, one can use the simplified expression given by (37) instead of the complete equation given by (39). With this simplification, the vector potential $\psi$ is given by equations (7) and (37). This describes the localized solutions of equations (5) that we are looking for.

\section{Study of localized solutions}

First of all, let us notice that for obtaining the equations for variables $\xi_{1}, \xi_{2} \ldots$ in explicit form, it is convenient to use (18). ¿From this equation, one can indeed analytically calculate the integrals that define the solutions. Taking into account the analysis presented in previous sections, the expressions (7) and (37) can then be used as a first order approximation of the solutions in the domain $\left|z c_{0}\right| \leqslant 1$ (note that the singularity occurs at $z c_{0}=1.18>$, thus outside this domain). These solutions are characterized by three dimensionless constants $c_{0}, M_{0}, B_{1}$. They are of the first order in $\epsilon^{2}$. $B_{1}$ is always positive, $M_{0}$ can be either positive or negative and, the function $\Psi$ (7) being an even function of $c$, the sign of $c_{0}$ has no importance. We can thus assume: $c_{0}>0$. The constant $B_{1}$ defines the characteristic space scale of the localized solution in dimensionless variables. For $\mathrm{x} \gg 2 \sqrt{B_{1}}$ the solution rapidly tends to the Harris type solution. $c_{0}$ is linked to the limit values of $\Psi$ as $x \rightarrow \infty$ on the boundary plasma surfaces. We will show later that $c_{0}$ is linked to the definition of the plasma density $n_{0}$, while $\left|M_{0}\right|$ determine the maximum value of the perpendicular component of the magnetic field $B_{Z}$.

\subsection{Isolated plasmoids and $\mathrm{X}$ points}

Let us rewrite (7) as:

$$
\frac{1}{\sqrt{2}} \exp \left(-\frac{\Psi}{2}\right)=\frac{c}{\cosh (z c)}
$$

By developing the R.H.S of (44) near the origin: $x=z=0$, one can show that, for $M_{0}>0$, the origin appears to be an elliptic point for the mapping of the magnetic field ( $\mathrm{O}$ - point). For $M_{0}<0$, the origin is an hyperbolic point which corresponds to an X-point mapping. The sign of $M_{0}$ then 
determines magnetic field configurations presenting different topologies. Examples corresponding to these two different topologies are given in figure 1 $\left(M_{0}=1,-1\right) \mathrm{ftbpFU} 4.5074 \mathrm{in} 6.173 \mathrm{in0pt}$ Examples of plasmoid and X-point configurations

The previous calculations have been performed using dimensionless variables. To make the connection with physical quantities, the following substitutions must be made :

$$
x \rightarrow \frac{a x}{L_{x}} ; z \rightarrow \frac{a z}{L_{z}}
$$

where $a$ is given by (80) in Appendix A. Using the vector potential formula and taking into account $(7),(37)$ and $(70)$, one finds the magnetic field component $B_{x}$ and $B_{z}$ :

$$
\begin{gathered}
B_{x}=-\frac{\left|u_{y i}\right|}{u_{y i}} \sqrt{8 \pi n_{0}\left(T_{i}+T_{e}\right)} \frac{\sqrt{2} c}{L_{z}} \tanh c \frac{a z}{L_{z}} \\
B_{z}= \pm \frac{\left|u_{y i}\right|}{u_{y i}} \sqrt{8 \pi n_{0}\left(T_{i}+T_{e}\right)} \frac{\varepsilon^{2}\left|M_{0}\right|}{L_{x}\left(2 B_{1}\right)^{3 / 2} c} \frac{a x}{L_{x}} \exp \left(-\frac{(a x)^{2}}{4 B_{1} L_{x}^{2}}\right)\left[1-c \frac{a z}{L_{z}} \tanh c \frac{a z}{L_{z}}\right]
\end{gathered}
$$

The plasma density is obtained from formula (74):

$$
n(x, z)=\frac{n_{0}}{L_{z}^{2}} \frac{2 c^{2}}{\cosh ^{2}\left(c \frac{a z}{L_{z}}\right)}
$$

Where $n_{0}$ is the plasma density in the Harris layer center $(z=0)$ for $x \rightarrow \pm \infty$. From (48), one gets:

$$
\frac{L_{z}}{\sqrt{2}}=c_{0}
$$

So $c_{0}$ defines the plasma layer thickness in dimensionless variables. The magnetic field $B_{0}$ verifies

$$
\frac{B_{0}^{2}}{8 \pi}=n_{0}\left(T_{i}+T_{e}\right)
$$

Then, the magnetic field $B_{x}$ can be written as:

$$
B_{x}=-B_{0} \tanh \left(\frac{c a z}{c_{0} \sqrt{2}}\right)
$$


The plasma density takes the form :

$$
n(x, z)=n_{0}\left(\frac{c}{c_{0}}\right)^{2} \frac{1}{\cosh ^{2}\left(\frac{c a z}{c_{0} \sqrt{2}}\right)}
$$

The parameter $a$ being given by the formula :

$$
a=\frac{B_{0}|e| u_{y i}}{c T_{i} \sqrt{2}}=\frac{2}{\rho_{B_{0} i}}\left(\frac{u_{y i}}{V_{T_{i}}}\right)
$$

where: $\quad \rho_{B_{0} i}$ is Larmor radius of ions in the magnetic field $B_{0}$,

$V_{T i}$ is the thermal velocity of ions, and

$u_{y i}$ is their current (diamagnetic) velocity.

It is clear from (85), that $a$ determines the Harris layer thickness $L_{H}$ :

$$
L_{H}=\frac{\rho_{B_{0} i}}{\sqrt{2}} \frac{V_{T_{i}}}{u_{y i}}
$$

As it follows from (80), $\left|M_{0}\right|$ determines the maximum value of the $B_{z}$ component :

$$
\left|B_{z}\right|=B_{0} \frac{a^{2}}{L_{x} L_{z}} \frac{\left|M_{0}\right|}{(2 B)^{3 / 2}}
$$

and the difference between plasma density in plasmoid center and $n_{0}$, see (51):

$$
\frac{n(0)-n_{0}}{n_{0}}= \pm \varepsilon^{2} \frac{\left|M_{0}\right|}{2 c_{0} \sqrt{B_{1}}}
$$

The characteristic horizontal scale $L_{x}$ of the structure is :

$$
L_{\text {॥ }}=L_{x} \sqrt{B_{1}} \rho_{B_{0} i}\left(\frac{V_{T_{i}}}{u_{y i}}\right)
$$

In other words, the plasmoid has an elliptical form, the ratio of its typical horizontal and vertical scales being :

$$
\frac{L_{H}}{L_{\text {॥I }}} \approx \frac{1}{\sqrt{2 B_{1}} L_{x}} \ll 1
$$

as it was assumed earlier.

Concerning the plasma boundaries, one sees that with $|x| \rightarrow \infty, B_{z} \rightarrow 0$. One also gets : 


$$
\begin{gathered}
B_{x} \rightarrow-B_{0} \tanh \left(\frac{z}{L_{H}}\right) ; A_{y}=-\frac{c T_{i}}{|e| u_{y i}} \Psi \\
\Psi \rightarrow \ln \cosh ^{2}\left(\frac{z}{L_{H}}\right) .
\end{gathered}
$$

As a result, a given value $\max \left|B_{x}(\infty)\right| \lesssim B_{0}$ defines a plane $\left|z_{0}\right|=$ const. $\lesssim L_{H}$ that determines the plasma boundaries at infinity and the associated value of $\Psi$ :

$$
\Psi=\Psi_{0}=\ln \cosh ^{2}\left(\frac{z_{0}}{L_{H}}\right)
$$

This also defines the vector potential $A_{y}^{0}$ on the magnetic surface that bounds the plasma layer. In the absence of plasma in the exterior domain, $\Psi$ or $A_{y}^{e x t}$ obey to the 2-D Laplace equation :

$$
\Delta_{2} A_{y}^{e x t}=0
$$

and to the usual Grad-Shafranov boundary conditions at the magnetic surface that bounds the plasma sheet

$$
A_{y}^{e x t}=A_{y}^{0}=\text { Const. } ; \frac{d A_{y}^{e x t}}{d \mathbf{n}}=\frac{d A_{y}^{i n t}}{d \mathbf{n}}
$$

We do not examine in details the solution of the exterior problem. We simply note that the solution will have the following form :

$$
A_{y}^{e x t}=B_{0}\left(z-z_{0}\right) \tanh \left(\frac{z_{0}}{L_{H}}\right)+A_{y}^{0}\left(z_{0}\right)+\widetilde{A}_{y}(z, x, \varepsilon)
$$

where the function $\widetilde{A}_{y}(z, x, \varepsilon)$ increases like $\ln z$ for $z \gg z_{0}$ and tends to zero as $|x| \rightarrow \infty$ or $\varepsilon \rightarrow 0$. If the distance from the plasma boundaries increases then, the magnetic field tends to a constant value :

$$
B_{x}^{0}=-B_{0} \tanh \left(\frac{z_{0}}{L_{H}}\right)
$$




\subsection{Multiplasmoids}

More complex solutions can also be proposed. They correspond to multiple plasmoids separated by $\mathrm{X}$ points. Let us consider the more general solutions of the first approximation equation (31) and let us choose the following function for $u\left(x^{\prime}\right)$ :

$$
u\left(x^{\prime}\right)=M_{0} \sqrt{\pi} \sum_{i=1}^{N} b_{i} \delta\left(x^{\prime}-x_{0 i}\right)
$$

where $\mathrm{b}_{i}$, and $\mathrm{x}_{0, i}$ are constant parameters. Then, instead of (32) we obtain :

$$
c_{2}=\frac{M_{0}}{2 \sqrt{\xi_{1}}} \sum_{i=1}^{N} b_{i} \exp \left[-\frac{\left(x^{\prime}-x_{0 i}\right)^{2}}{4 \xi_{1}}\right]
$$

where $\xi_{1}$ has the form given in (30). The solvability conditions for the terms of increasing order can be obtained in the same way as for the function (83), see Appendix B. In order to do it, we have to replace $c_{2}$ given by (30) and appearing in all formulae of the Appendix B by $c_{2}$ given by (66). The expression becomes more complex. Nevertheless, the general scheme presented in the Appendix B remains valid. New solutions are then obtained. Their mathematical expression can be obtained using equations (7) and

$$
c=c_{0}+\varepsilon^{2} \frac{M_{0}}{2 \sqrt{B_{1}}} \sum_{i=1}^{N} b_{i} \exp \left[-\frac{\left(x^{\prime}-x_{0 i}\right)^{2}}{4 B_{1}}\right]
$$

Some examples of magnetic field configurations obtained from this formula are presented in figure 2. Solution with $b_{i}>0$ describes a set of plasmoids with amplitudes $b_{i}$ and centered at $x_{0 i}$. This corresponds to a multiplasmoid solution. For arbitrary $b_{i}$ either negative or positive; multiplasmoid solutions presenting a more or less sharp transition to hyperbolic $\mathrm{X}$ points are obtained. ftbpFU4.5074in6.173in0ptExamples of multiplasmoid solutionsf $f_{p} l a s 2 . p s$

\section{Conclusion}

In this paper, we have proposed a new asymptotic scheme that allows to obtain in a systematic way approximative solutions for the 2-D "Harris" equation. This asymptotic development differs from the classic one proposed by K. Shindler by the treatment of the boundary conditions. In contrast to Schindler's work, we 
indeed analyze a free boundary situation or, more exactly, a situation for which the plasma boundaries are not determined in advance. The plasma will be then bounded by magnetic surfaces defined by the condition: $\Psi=$ Const.; the form of these surfaces being, however, uniquely determined by the solution itself and the magnetic field value at infinity .Out of the plasma, the vector potential can be deduced form the Laplace equation and, as usual for the Grad-Shafranov equations, from the continuity of $\Psi$ and its normal derivative at the plasma boundaries.

The main difficulty of the problem relies on the fact that one has to systematically eliminate the increasing and the singular terms that appear in the perturbation theory. From the elimination procedure, we found particular solutions that describe localized plasmoids embedded in the sheet. The simplest solution describing a plasmoid is characterized by three parameters : the magnetic field value at infinity, the typical horizontal scale and the plasma density in the center of the plasmoid (or more exactly, its deviation from its value in the center of the non-perturbed 1-D Harris layer). Another parameter is the maximum value of the perpendicular magnetic field component. The magnetic field in the plasmoid center is zero and presents a singularity of elliptical type. Out of plasma, the magnetic field is approximately constant. Both the pressure and the plasma density have their maximum in the center of the plasmoid. In the presence of orthogonal homogeneous electrical and magnetic fields, the system of equations remains valid and will describe solutions moving along the layer with the electrical drift velocity.

¿From the solvability conditions, another solution corresponding to $\mathrm{X}$ point magnetic field configurations has been obtained. As already mentioned, the use of the complete Maxwell-Vlasov equations instead of the ideal MHD equation guarantees the physical validity of this type of solutions. We also find more general solutions that describe sets of plasmoids (multiplasmoid) separated by $\mathrm{X}$ points.

The origin of the different topologies deserves some comments. With $\varepsilon^{2}=$ 0 ,the zero approximation equation describes a 1-D vector field. With $\varepsilon^{2} \neq 0$ the perturbed equation describes a 2 -D vector field in the plane $(z, x)$. It means that the perturbation does not conserve the dimensionality of the space and is not a homomorphism (it is well-known that only homomorphism saves space dimensionality). Since the perturbed equation describes a 2-D vector field in a plane, its typical singularity are elliptical and/or hyperbolic points and this is what was obtained from the asymptotic development.

Let us finally note that it is clear from (37) and (67) that we have only examined a simple case of particular solutions. As a matter of fact, a wider class of asymptotic solutions exists and depends on the form of the function $u\left(x^{\prime}\right)$. However, for these different cases, it has not been verified that it is actually possible to eliminate the increasing and singular terms in the next approximation. 


\section{Appendix A. Basic Equations}

Let us consider a plasma in a constant magnetic field $B_{y}^{0}$ and a constant electrical field $E_{z}^{0}$, the system being invariant by translation along the $y$ axis. We can choose the vector potential $\mathbf{A}$ in following form :

$$
\mathbf{A}=\left(A_{x}(x, z) ; A_{y}(x, z) ; 0\right)
$$

Taking into account the Coulomb gauge :

$$
\frac{\partial A_{x}}{\partial x}=0, \Longrightarrow A_{x}=A_{x}(z)
$$

Since $B_{y}^{0}=$ const,

$$
B_{y}^{0}=\frac{\partial A_{x}}{\partial z}=\text { Const }, \Longrightarrow A_{x}=B_{y}^{0} z
$$

The magnetic field writes :

$$
\mathbf{B}=\operatorname{rot} \mathbf{A}=\left(-\frac{\partial A_{y}(x, z)}{\partial z}\right) \mathbf{i}+B_{y}^{0} \mathbf{j}+\left(\frac{\partial A_{y}(x, z)}{\partial x}\right) \mathbf{k}
$$

since

$$
\mathbf{B} \nabla A_{y}(x, z)=0
$$

then the magnetic field lines are located on the magnetic surfaces $A_{y}(x, z)=$ $\Psi(x, z)=$ const.

¿From the y-invariance follows the conservation of canonical momentum $P_{y}$.

$$
P_{y}=m V_{y}+\frac{e}{c} A_{y}
$$

Let us note here that if the plasma moves along the sheet with a constant velocity $u_{x}=u_{0}$, the time will appear through the variable $\xi=x-u_{0} t$ only.

The Lagrange function of non relativistic particles $£$ and energy $\mathcal{E}$ write:

$$
\begin{gathered}
£=\frac{m V^{2}}{2}+\frac{e}{q} \mathbf{V A}-e \Phi \\
\mathcal{E}=\frac{m V^{2}}{2}+e \Phi
\end{gathered}
$$


where $\Phi$ - is a scalar potential

$$
\Phi=-E_{z}^{0} z
$$

¿From the time dependance of the energy:

$$
\frac{d \mathcal{E}}{d t}+\frac{\partial £}{\partial t}=0
$$

and the Lagrange equation, we obtain :

$$
\frac{d}{d t}\left(\mathcal{E}-u_{0} \frac{\partial £}{\partial \dot{x}}\right)=0
$$

A second invariant $W$ is then :

$$
W=\frac{m V^{2}}{2}+e \Phi-u_{0}\left(m V_{x}+\frac{e}{c} A_{x}(z)\right)
$$

Since stationary solutions of the Vlasov equation are function of these two invariants (71) and (72), one gets :

$$
f=f_{0}\left(P_{y}, W\right)
$$

Choosing as usual $f_{0}$ as a Maxwell distribution function, we obtain the usual Harris distribution function [7]:

$$
\begin{aligned}
f_{0 j} & =n_{0 j}() m_{j} 2 \pi T_{j}^{\frac{3}{2}} \exp \left(\frac{u_{y j}-W_{j}}{T_{j}}\right)= \\
& =n_{j}(x, z)() m_{j} 2 \pi T_{j}^{\frac{3}{2}} \exp \left\{-\frac{m_{j}}{2 T_{j}}\left[\left(v_{x}-u_{0}\right)^{2}+\left(v_{y}-u_{y j}\right)^{2}+v_{z}^{2}\right]\right\}
\end{aligned}
$$

Here:

$$
n_{j}(x, z)=n_{0 j} \exp \left(\frac{q_{j} u_{y j} A_{y}}{c T_{j}}\right) \exp \left(q_{j} \frac{u_{0} A_{x}-c \Phi}{c T_{j}}\right)
$$

where $u_{y j}$ correspond to the velocity of the current and the $j$ subscript to the electrons and the ions $(j=e, i)$. The quasi- neutrality condition gives [7]:

$$
n_{0 e}=n_{0 j} ; \frac{u_{y e}}{T_{e}}+\frac{u_{y i}}{T_{i}}=0
$$

and the additional conditions: 


$$
\begin{gathered}
-u_{0} A_{x}(z)+c \Phi(z)=0 ; \Phi_{z z}^{\prime \prime}=0 \\
\Phi(z)=-E_{z}^{0} z ; A_{x}(z)=B_{y}^{0} z ; \\
u_{0}=-\frac{c E_{z}^{0}}{B_{y}^{0}} ;
\end{gathered}
$$

where $u_{0}$ is obviously the electrical drift velocity.

Taking into account the quasi neutrality conditions (75), the plasma pressure for the Harris distribution function is:

$$
P=n_{0}\left(T_{e}+T_{i}\right) \exp \left(\frac{e_{i} u_{y i} A_{y}}{c T_{i}}\right)
$$

Maxwell equations give :

$$
\begin{gathered}
r o t \mathbf{B}=\frac{4 \pi}{c} \mathbf{J}+\frac{1}{c} \frac{\partial \mathbf{E}}{\partial t}=\frac{4 \pi}{c} \mathbf{J}-\frac{u_{0}}{c} \frac{\partial \mathbf{E}}{\partial x} \\
\mathbf{E}=-\frac{1}{c} \frac{\partial \mathbf{A}}{\partial t}+\mathbf{k} E_{z}^{0} ;
\end{gathered}
$$

As a result, we obtain the equation :

$$
\frac{4 \pi}{c} J_{y}=-\left[\frac{\partial^{2} A_{y}}{\partial x^{2}}+\left(1-\frac{u_{0}^{2}}{c^{2}}\right) \frac{\partial^{2} A_{y}}{\partial x^{2}}\right]
$$

Since we study a non relativistic case $\frac{u_{0}^{2}}{c^{2}} \ll 1$, we can omit the term containing $\frac{u_{0}^{2}}{c^{2}}$. Calculating the current $j_{y}$ by using the Harris distribution function(77), the Ampere equation takes the form :

$$
-\left(\frac{\partial^{2} A_{y}}{\partial x^{2}}+\frac{\partial^{2} A_{y}}{\partial x^{2}}\right)=\frac{4 \pi n_{0}|e|}{c} u_{y i}\left(1+\frac{T_{e}}{T_{i}}\right) \exp \left(\frac{e_{i} u_{y i} A_{y}}{c T_{i}}\right)
$$

Then, using dimensionless variables :

$$
A_{y}=-\frac{c T_{i}}{|e| u_{y i}} \Psi ; x^{\prime}=a x ; z^{\prime}=a z
$$




$$
a=\frac{\sqrt{4 \pi n_{0}}\left|e u_{y i}\right|}{c} \frac{\sqrt{T_{e}+T_{i}}}{T_{i}}=\sqrt{2} \frac{\varpi_{p i}}{c} \frac{\left|u_{y i}\right|}{v_{T_{i}}} \sqrt{\frac{T_{e}+T_{i}}{T_{i}}}
$$

The equation (1) ("Harris" equation) is obtained.

In the rest of the paper, we will omit primes for dimensionless variables $x^{\prime}, z^{\prime}$. The magnetic field $\mathbf{B}$ is expressed by means of $\Psi$ :

$$
\begin{aligned}
& B_{x}=-2 \sqrt{\pi n_{0}\left(T_{e}+T_{i}\right)} \frac{\left|u_{y i}\right|}{u_{y i}} \frac{\partial \Psi}{\partial z} \\
& B_{z}=+2 \sqrt{\pi n_{0}\left(T_{e}+T_{i}\right)} \frac{\left|u_{y i}\right|}{u_{y i}} \frac{\partial \Psi}{\partial x}
\end{aligned}
$$

Considering again the Ampere equation (78), we can multiply equation (78) by $\frac{\partial A_{y}}{\partial x}$ and then $\frac{\partial A_{y}}{\partial z}$. The resulting equations are clearly the $x$ and $z$ components of the MHD equation:

$$
\frac{1}{4 \pi}\left[\operatorname{rot} \mathbf{B}\left(A_{y}\right) \times \mathbf{B}\left(A_{y}\right)\right]=\nabla P\left(A_{y}\right)
$$

where the pressure $P\left(A_{y}\right)$ in (82) is defined by the Harris function (77).Thus, for this particular case, the Ampere equation (78) and the MHD equilibrium equation (82) coincide formally and correspond to the Grad-Shafranof equations. They must be solved in plasma domain confined by magnetic surfaces $\Psi=$ const. Outside this domain, the plasma is absent and the vector potential $\Psi$ has to be deduced from the Laplace equation and boundary conditions corresponding to the continuity of $\Psi$ and $\frac{\partial \Psi}{\partial \mathbf{n}}$ at the plasma-vacuum boundary .

\subsection{Appendix B. Elimination of increasing terms in $\varepsilon^{6}$ ap- proximation}

Let us put the asymptotic development (22) and the expression (24) in the equation (8) and write together the different terms of order $\varepsilon^{6}$. We will consider $M_{0}$ as a function of $\xi_{2}, \tilde{\xi}_{2}$, then the first approximation has the form :

$$
c_{2}=\frac{M_{0}\left(\xi_{2}, \tilde{\xi_{2}}\right)}{2 \sqrt{\xi_{1}}} \exp \left(-\frac{x^{2}}{4 \xi_{1}}\right)
$$

To met further solvability conditions we will find $M_{0}\left(\xi_{2}, \widetilde{\xi_{2}}\right)$ in factorized form 


$$
M_{0}\left(\xi_{2}, \tilde{\xi}_{2}\right)=M_{01}\left(\xi_{2}\right) M_{02}\left(\tilde{\xi}_{2}\left(\xi_{2}, x, z\right)\right)
$$

After dividing equation by $f_{0}$, we write the resulting equation in the following form :

$$
\left(\frac{2 f_{0 z}^{\prime}}{f_{0}} F_{1}^{\prime}+F_{1}^{\prime \prime}\right) \frac{\partial c_{4}}{\partial \xi_{1}}+\frac{\partial^{2} c_{4}}{\partial x^{2}}+\frac{N\left[c_{2}\right]}{f_{0}}+\frac{L\left[c_{2}\right]}{f_{0}}=0
$$

Here, $L\left[c_{2}\right]$ denotes the linear terms in $c_{2}$ and $N\left[c_{2}\right]$ the non-linear ones:

$$
\begin{aligned}
& \frac{L\left[c_{2}\right]}{f_{0}}=\left(\frac{2 f_{0 z}^{\prime}}{f_{0}} F_{1}^{\prime}+F_{1}^{\prime \prime}\right) \frac{1}{2 \sqrt{\xi_{1}}} \exp \left(-\frac{x^{2}}{4 \xi_{1}}\right) \frac{\partial M_{0}}{\partial \xi_{2}}+ \\
&+\left(\frac{2 f_{0 z}^{\prime}}{f_{0}} \tilde{F}_{2}^{\prime}+\tilde{F}_{2}^{\prime \prime}\right) \frac{1}{2 \sqrt{\xi_{1}}} \exp \left(-\frac{x^{2}}{4 \xi_{1}}\right) \frac{\partial M_{0}}{\partial \tilde{\xi}_{2}}+ \\
&+\left(F_{1}^{\prime}\right)^{2} \frac{M_{0}}{2} \frac{\partial^{2}}{\partial \xi_{1}^{2}} \frac{1}{\sqrt{\xi_{1}}} \exp \left(-\frac{x^{2}}{4 \xi_{1}}\right) ; \\
& {\left[\frac{1}{2 \sqrt{\xi_{1}}} \exp \left(-\frac{x^{2}}{4 \xi_{1}}\right)\right]^{-1} \frac{L\left[c_{2}\right]}{f_{0}}=\left(\frac{2 f_{0 z}^{\prime}}{f_{0}} F_{2}^{\prime}+F_{2}^{\prime \prime}\right) \frac{\partial M_{0}}{\partial \xi_{2}}+} \\
&+\left(\frac{2 f_{0 z}^{\prime}}{f_{0}} F_{2}^{\prime}+F_{2}^{\prime \prime}\right) \frac{\partial M_{0}}{\partial \xi_{2}}+\left(\frac{2 f_{0 z}^{\prime}}{f_{0}} \tilde{F}_{2}^{\prime}+\tilde{F}_{2}^{\prime \prime}\right) \frac{\partial M_{0}}{\partial \tilde{\xi}_{2}}+ \\
&+M_{0}\left(F_{1}^{\prime}\right)^{2}\left(\frac{3}{4 \xi_{1}^{2}}-\frac{3 x^{2}}{4 \xi_{1}^{3}}+\frac{x^{4}}{16 \xi_{1}^{4}}\right)
\end{aligned}
$$

$\frac{N\left[C_{2}\right]}{f_{0}}$ contains several groups of terms:

$$
\frac{N\left[C_{2}\right]}{f_{0}}=\frac{N_{1}\left[C_{2}\right]}{f_{0}}+\frac{N_{2}\left[C_{2}\right]}{f_{0}}+\frac{N_{3}\left[C_{2}\right]}{f_{0}}
$$

$\frac{N_{1}\left[C_{2}\right]}{f_{0}}$ is the contribution from the first term in the equation (9), $\frac{N_{2}\left[C_{2}\right]}{f_{0}}$ the contribution from the second term in (9) and, $\frac{N_{3}\left[C_{2}\right]}{f_{0}}$ the contribution from the left part of the equation (8).

$$
\begin{aligned}
\frac{N_{1}\left[C_{2}\right]}{f_{0}} & =\frac{2}{c_{0}^{2}} \frac{\left(z c_{0}\right)^{2}+\cosh ^{2} z c_{0}}{f_{0} \cosh ^{2} z c_{0}}\left(\frac{\partial c_{2}}{\partial x}\right)^{2}= \\
& =-\frac{M_{0}^{2} x^{2}}{16 \xi_{1}^{3} c_{0}} \exp \left(-\frac{x^{2}}{2 \xi_{1}}\right) \frac{\left(z c_{0}\right)^{2}+\cosh ^{2} z c_{0}}{\left(1-z c_{0} \tanh z c_{0}\right) \cosh ^{2} z c_{0}}
\end{aligned}
$$




$$
\begin{gathered}
\frac{N_{2}\left[C_{2}\right]}{f_{0}}=\frac{1}{f_{0}} \frac{\partial f_{0}}{\partial c_{0}} c_{2} \frac{\partial^{2} c_{2}}{\partial x^{2}}= \\
=\frac{M_{0}}{\left(1-z c_{0} \tanh z c_{0}\right)}\left[\frac{\left(z c_{0}\right)^{2}+\cosh ^{2} z c_{0}}{\cosh ^{2} z c_{0}}\right] \exp \left(-\frac{x^{2}}{2 \xi_{1}}\right) \frac{\left(\frac{x^{2}}{2 \xi_{1}}-1\right)}{8 c_{0} \xi_{1}^{2}} \\
\frac{N_{3}\left[C_{2}\right]}{f_{0}}=\frac{1}{f_{0}}\left[2\left(\frac{\partial f_{0 z}^{\prime}}{\partial c_{0}}\right) F_{1}^{\prime}+\left(\frac{\partial f_{0}}{\partial c_{0}}\right) F_{1}^{\prime \prime}\right] c_{2} \frac{\partial c_{2}}{\partial \xi_{1}}= \\
=-\frac{M_{0}^{2}}{8 \xi_{1}^{2} \cosh ^{2} z c_{0}}\left[4 z c_{0} F_{1}^{\prime}+\frac{\left(z c_{0}\right)^{2}+\cosh ^{2} z c_{0}}{c_{0}\left(1-z c_{0} \tanh ^{2} z c_{0}\right)} F_{1}^{\prime \prime}\right] \exp \left(-\frac{x^{2}}{2 \xi_{1}}\right)\left(\frac{x^{2}}{2 \xi_{1}}-1\right)
\end{gathered}
$$

Taking into account (27) with $\lambda=1,(85)$ takes the form :

$$
\frac{\partial C_{4}}{\partial \xi_{1}}-\frac{\partial^{2} C_{4}}{\partial x^{2}}=\frac{N\left[C_{2}\right]}{f_{0}}+\frac{L\left[C_{2}\right]}{f_{0}}
$$

As we can see from (87) -(90), the right part contains a singularity at $\left(z c_{0}\right) \sim$ 1 which lead to increasing terms. In order to eliminate them, we have to require the right part to be equal to zero. In this case, for $c_{4}$, we can take simplest solutions as in section 2.2 :

$$
c_{4}=\frac{M_{2}}{2 \sqrt{\xi_{1}}} \exp \left(-\frac{x^{2}}{4 \xi_{1}}\right)
$$

When the right hand part of (91) is zero, we obtain an equation that can be considered as the solvability condition :

$$
\begin{aligned}
& 0=M_{02}\left(\tilde{\xi_{2}}\right)\left[\left(\frac{2 f_{0 z}^{\prime}}{f_{0}} F_{2}^{\prime}+F_{2}^{\prime \prime}\right) \frac{\partial M_{01}\left(\xi_{2}\right)}{\partial \xi_{2}}+M_{01}\left(\xi_{2}\right)\left(F_{1}^{\prime}\right)^{2}\left(\frac{3}{4 \xi_{1}^{2}}-\frac{3 x^{2}}{4 \xi_{1}^{3}}+\frac{x^{4}}{16 \xi_{1}^{4}}\right)\right]+ \\
& +M_{01}\left(\xi_{2}\right)\left(\frac{2 f_{0 z}^{\prime}}{f_{0}} \tilde{F}_{2}^{\prime}+\tilde{F}_{2}^{\prime \prime}\right) \frac{\partial M_{02}\left(\tilde{\xi_{2}}\right)}{\partial \tilde{\xi}_{2}}-\frac{M_{01}^{2}\left(\xi_{2}\right) M_{02}^{2}\left(\tilde{\xi_{2}}\right)}{8 c_{0} \xi_{1}^{2} \sqrt{\xi_{1}}} \exp \left(-\frac{x^{2}}{4 \xi_{1}}\right) \frac{x^{2}}{\left(1-z c_{0} \tanh z c_{0}\right)}\left[\frac{\left(z c_{0}\right)^{2}}{\mathrm{cc}}\right. \\
& +\frac{M_{01}^{2}\left(\xi_{2}\right) M_{02}^{2}\left(\tilde{\xi_{2}}\right)}{4 c_{0} \xi_{1} \sqrt{\xi_{1}}} \exp \left(-\frac{x^{2}}{4 \xi_{1}}\right) \frac{\left(\frac{x^{2}}{2 \xi_{1}}-1\right)}{\left(1-z c_{0} \tanh z c_{0}\right)}\left[\frac{\left(z c_{0}\right)^{2}+\cosh ^{2} z c_{0}}{\cosh ^{2} z c_{0}}\right]- \\
& -\frac{M_{01}^{2}\left(\xi_{2}\right) M_{02}^{2}\left(\tilde{\xi}_{2}\right)}{4 \xi_{1} \sqrt{\xi_{1}}} \exp \left(-\frac{x^{2}}{4 \xi_{1}}\right) \frac{\left(\frac{x^{2}}{2 \xi_{1}}-1\right)}{\cosh ^{2} z c_{0}}\left[4 z c_{0} F_{1}^{\prime}+\frac{\left(z c_{0}\right)^{2}+\cosh ^{2} z c_{0}}{c_{0}\left(1-z c_{0} \tanh z c_{0}\right)} F_{1}^{\prime \prime}\right]
\end{aligned}
$$


We shall note that the term :

$$
\frac{\partial \tilde{\xi}_{2}}{\partial \xi_{2}}=\varepsilon^{2} \frac{\partial \tilde{F}_{2}\left(\xi_{2}\right)}{\partial \xi_{2}}
$$

belong to the higher order equations. Let us define the $F_{2}$ function by the equation :

$$
\left(\frac{2 f_{0 z}^{\prime}}{f_{0}} F_{2}^{\prime}+F_{2}^{\prime \prime}\right)=\left(F_{1}^{\prime}\right)^{2}\left(\frac{3}{4 \xi_{1}^{2}}-\frac{3 x^{2}}{4 \xi_{1}^{3}}+\frac{x^{4}}{16 \xi_{1}^{4}}\right)
$$

For the function $M_{01}\left(\xi_{2}\right)$, we obtain from (93):

$$
\frac{\partial M_{01}\left(\xi_{2}\right)}{\partial \xi_{2}}+M_{01}\left(\xi_{2}\right)=0
$$

This is the vanishing condition for the coefficient of $M_{02}\left(\tilde{\xi}_{2}\right)$ in (93). As follows from (95) :

$$
M_{01}\left(\xi_{2}\right)=A \exp \left(-\xi_{2}\right) .
$$

( $A=$ const.) From (94), one can find the function $F_{2}$ :

$$
F_{2}=\int \frac{d z}{f_{0}^{2}} \int f_{0}^{2}\left(F_{1}^{\prime}\right)^{2}\left(\frac{3}{4 \xi_{1}^{2}}-\frac{3 x^{2}}{4 \xi_{1}^{3}}+\frac{x^{4}}{16 \xi_{1}^{4}}\right) d z
$$

This integral will be calculated approximately as the integral (28). When calculating integral (97) we shall take into account only the contribution from the leading singularity. Others factors will be put out of the integral because they are changing slowly when $0 \leq\left(z c_{0}\right)^{2} \lesssim 1-\varepsilon^{2}$.

$$
\begin{aligned}
& F_{2} \simeq \frac{1}{4 \xi_{1}^{2}}\left(\frac{3}{4 \xi_{1}^{2}}-\frac{3 x^{2}}{4 \xi_{1}^{3}}+\frac{x^{4}}{16 \xi_{1}^{4}}\right) \int \frac{d z}{f_{0}^{2}} \int f_{0}^{2}\left(F_{1}^{\prime}\right)^{2} d z= \\
= & \frac{1}{2 c_{0}^{4} 15^{2} \xi_{1}^{2}}\left(\frac{3}{\xi_{1}^{2}}-\frac{3 x^{2}}{\xi_{1}^{3}}+\frac{x^{4}}{4 \xi_{1}^{4}}\right)\left[\frac{1}{\left(1-z c_{0}\right)^{2}}+\frac{1}{\left(1+z c_{0}\right)^{2}}\right]
\end{aligned}
$$

As a result we obtain for $\xi_{2}$ :

$$
\xi_{2} \simeq \frac{\varepsilon^{4}}{c_{0}^{4} 15^{2} \xi_{1}^{2}} \frac{\left[1+\left(z c_{0}\right)^{2}\right]}{\left[1-\left(z c_{0}\right)^{2}\right]^{2}}\left(\frac{3}{\xi_{1}^{2}}-\frac{3 x^{2}}{\xi_{1}^{3}}+\frac{x^{4}}{4 \xi_{1}^{4}}\right)+B_{2}
$$


Let us now define the function $\widetilde{F}_{2}$ by the equation :

$$
\begin{aligned}
& H\left(\frac{2 f_{0 z}^{\prime}}{f_{0}} \tilde{F}_{2}^{\prime}+\tilde{F}_{2}^{\prime \prime}\right)=-\frac{x^{2}}{8 c_{0} \xi_{1}^{2}}\left[\frac{\left(z c_{0}\right)^{2}+\cosh ^{2} z c_{0}}{\left(1-z c_{0} \tanh z c_{0}\right) \cosh ^{2} z c_{0}}\right]+ \\
& +\left[\frac{\left(z c_{0}\right)^{2}+\cosh ^{2} z c_{0}}{4 c_{0} \xi_{1}\left(1-z c_{0} \tanh z c_{0}\right) \cosh ^{2} z c_{0}}\right]\left(\frac{x^{2}}{2 \xi_{1}}-1\right)- \\
& -\frac{1}{4 \xi_{1} \cosh ^{2} z c_{0}}\left[4 z c_{0} F_{1}^{\prime}+\frac{\left(z c_{0}\right)^{2}+\cosh ^{2} z c_{0}}{c_{0}\left(1-z c_{0} \tanh z c_{0}\right)} F_{1}^{\prime \prime}\right]\left(\frac{x^{2}}{2 \xi_{1}}-1\right) \\
& H=\left[\mp \frac{1}{\sqrt{\xi_{1}}} \exp \left\{B_{2}-\frac{\varepsilon^{4}}{c_{0}^{4} 15^{2}} \frac{\left[1+\left(z c_{0}\right)^{2}\right]}{\left[1-\left(z c_{0}\right)^{2}\right]^{2}}\left(\frac{3}{\xi_{1}^{2}}-\frac{3 x^{2}}{\xi_{1}^{3}}+\frac{x^{4}}{4 \xi_{1}^{4}}\right)-\frac{x^{2}}{4 \xi_{1}}\right\}\right]^{-1}
\end{aligned}
$$

Then for $M_{02}\left(\tilde{\xi_{2}}\right)$, we obtain the equation :

$$
\frac{d M_{02}^{2}\left(\tilde{\xi_{2}}\right)}{d \tilde{\xi_{2}}} \pm M_{02}^{2}\left(\tilde{\xi_{2}}\right)=0
$$

The solution of (101) has obviously the form :

$$
M_{02}\left(\tilde{\xi_{2}}\right)=\frac{( \pm 1)}{\tilde{\xi_{2}}+D}
$$

In equations (101) and (100) two signs $( \pm)$ are possible. As earlier, we solve the equation (100) approximately. The main contribution in the integrals gives the stronger singularity in the right hand part of the equation (100) (the term with $\left.F^{\prime \prime}\right)$. Other terms correspond to weaker singularities when $0 \leq\left(z c_{0}\right)^{2} \lesssim$ $1-\varepsilon^{2}$. Again, slowly varying terms will be put out of the integral and thus, we obtain the approximative formulae :

$$
\begin{aligned}
\tilde{\xi}_{2} \simeq & \mp \frac{\varepsilon^{4}}{c_{0}^{4} 15^{2}} \frac{\left[1+\left(z c_{0}\right)^{2}\right]}{\left[1-\left(z c_{0}\right)^{2}\right]^{2}}\left(\frac{x^{2}}{2 \xi_{1}}-1\right) \frac{1}{\xi_{1} \sqrt{\xi_{1}}} \frac{\left(z c_{0}\right)^{2}+\cosh ^{2} z c_{0}}{\cosh ^{2} z c_{0}} \times \\
& \times \exp \left\{-B_{2}-\frac{x^{2}}{4 \xi_{1}}-\frac{\varepsilon^{4}}{c_{0}^{4} 15^{2}} \frac{\left[1+\left(z c_{0}\right)^{2}\right]}{\left[1-\left(z c_{0}\right)^{2}\right]^{2}}\left(\frac{3}{\xi_{1}^{2}}-\frac{3 x^{2}}{\xi_{1}^{3}}+\frac{x^{4}}{4 \xi_{1}^{4}}\right)\right\}+\tilde{B}_{2}
\end{aligned}
$$


Formulae (96),(99),(102) and, (103) are valid in the domain $0 \leq\left(z c_{0}\right)^{2} \lesssim 1-\varepsilon^{2}$ as well as formulae (30) and,(34). The formulae (96)and (102) together with (99) and (103) allow to find the coefficient $M_{0}$ (84). This value $M_{0}$ eliminates increasing terms in the next approximation. Finally, the first approximation solutions are given by formulae (39) and (41)in section 2.2.

\section{References}

[1] Parker,E.N.," Spontaneous Current Sheets in Magnetic Fields",Oxford Univ.Press.N.Y.,1999.

[2] Hones,E.W.,ed.," Magnetic Reconnection in Space and Laboratory Plasmas",Geophys.Monogr.Ser.vol.30,AGU,

Washington,D.C.,1984.

[3] Russell,C.T.,Priest,E.R.,and Lee,L.C. eds." Physics of Magnetic Flux Ropes",Geophys.Monogr.Ser.vol.58,AGU,

Washington,D.C.,1990.

[4] Biskamp,D ."Nonlinear Magnetohydrodynamics",Cambridge,Univ.Press.,N.Y.,1993.

[5] Slavin,J.A.,et al.,ISTP observations of plasmoid ejection:IMP 8 and Geotail,J.Geophys.Res.,103,119,1998.

[6] Lin,N.,McPherron,R.,L.,Kivelson,M.G.,and Walker,R.J.,Multipoint reconnection in the near-Earth magnetotail:CDAW6 observations of energetic particles and magnetic field,J.Geophys.Res.,v.96,p.19,427-19,439,1991.

[7] Harris,E.G.,On a plasma sheet separating regions of oppositely directed magnetic fields,Nuovo Cimento,23,115-121,1962.

[8] Amann,H.,Fixed point equation and nonlinear eigenvalue problems in ordered Banach spaces,SIAM Rev.,18,620,1976.

[9] Nevanlinna,R.,Die Einheit Analytische Funktionen,Berlin,1934.

[10] Fadeev,V.M.,Kvarskhava,I.F.,and Komarov,N.N.,Self-focusing of localized plasma currents,Nucl.Fusion, $5,202,1965$.

[11] Evgrafov,M.A., Assymptotical estimations and entire functions, M., "Nauka", 1979, (in russian).

[12] Schindler,K.,A Self-Consistent theory of the Tail of the Magnetosphere,in"Earth's magnetospheric processes", ed. by B.M.McCormac,p.200-209,D.Reidel,Norwood,Massachusetts,1972 . 
[13] Zwingmann,W.,Self consistent magnetotail theory:equilibrium structures including arbitrary variation along the tail axis,J.Geophys.Res.,v.88,p.9101-9108,1983.

[14] Birn,J.,Sommer,R.,and Schindler,K.,Open and closed magnetospheric tail configuration and their stability, Astrophys.and Space Science 35,p.389402,1975.

[15] Kivelson,M.G.,and Khurana,K.K.,Models of flux ropes embedded in a Harris neutral sheet:Force-free solutions in low and high beta plasmas,J.Geophys.Res.,A12,p.23,637-23,645,1995.

[16] Lembège,B.,and Pellat,R.,Stability of a thick two-dimensional quasineutral sheet,Phys.Fluids,25(11),p.1995-2004,1982.

[17] Voigt,G.H.,and Moore,B.D.,A Note on Two-dimensional Asimptotic Magnetotail Equilibria, J.Geophys.Res, v.99,p.5943-5948,1994.

[18] Wang,X.,and Bhattacharjee,A.,An Equilibrium Model of the Earth's Magnetotail:Thin Current Sheet and a near-Earth neutral line,J.Geophys.Res.,v.104,p.7045-7048,1999.

[19] Nayfeh,A.H.,Perturbation Methods,A Wiley-Interscience Publication,N.Y.,London, Toronto,1973.

[20] Lomov, S.A., Introduction in general theory of singular perturbation, M., "Nauka", 1981, (in russian). 